\title{
Patients' experiences of asthma exacerbation and management: a qualitative study of severe asthma
}

\author{
Woo-Jung Song $\mathbb{1}^{1}$, Ha-Kyeong Won ${ }^{2}$, Suh Young Lee ${ }^{3}$, Han-Ki Park $\mathbb{1}^{4}$, \\ You Sook Cho ${ }^{1}$, Kian Fan Chung ${ }^{5}$, Liam G. Heaney ${ }^{6}$ and Woo Joung Joung ${ }^{7}$
}

Affiliations: ${ }^{1}$ Dept of Allergy and Clinical Immunology, University of Ulsan College of Medicine, Asan Medical Center, Seoul, Korea. ${ }^{2}$ Dept of Internal Medicine, Veterans Health Service Medical Center, Seoul, Korea. ${ }^{3}$ Dept of Internal Medicine, Seoul National University College of Medicine, Seoul, Korea. ${ }^{4}$ Dept of Allergy and Clinical Immunology, Kyungpook National University Chilgok Hospital, School of Medicine, Kyungpook National University, Daegu, Korea. ${ }^{5}$ National Heart and Lung Institute, Imperial College London \& Royal Brompton and Harefield NHS Trust, London, UK. ${ }^{6}$ Centre for Experimental Medicine, School of Medicine, Dentistry and Biomedical Sciences, Queen's University Belfast, Belfast, UK. ${ }^{7}$ College of Nursing, Research Institute of Nursing Science, Kyungpook National University, Daegu, Korea.

Correspondence: Woo Joung Joung, College of Nursing, Kyungpook National University, Gukchebosangro, Jung-gu, Daegu, Korea, 41944. E-mail: godblessedaknu.ac.kr

ABSTRACT Exacerbation is a defining feature of severe asthma, and oral corticosteroids (OCSs) are frequently prescribed to manage exacerbations. This qualitative study was conducted to examine the experience of patients with severe asthma, with a focus on asthma exacerbation and OCS treatment.

Adults with severe asthma were recruited from three tertiary hospitals in South Korea. Data were collected through in-depth qualitative interviews. Verbatim transcripts were analysed using Colaizzi's phenomenological method to uncover the meaning of the participants' experience. Recruitment of participants continued until thematic saturation.

14 patients with severe asthma were recruited. Four theme clusters emerged: 1) experience of asthma exacerbation; 2) impact on life; 3) OCS treatments; and 4) disease perception. The patients experienced severe physical and psychosocial distress from asthma exacerbations, felt helpless due to failed efforts to prevent exacerbation and were living a restricted life due to fear of exacerbation. They feared OCS sideeffects but had no other choice than to rely on OCSs because other interventions were ineffective. Most had poor knowledge and understanding of severe asthma and the long-term health consequences.

Asthma exacerbation affects wide aspects of life in patients with severe asthma. Several components may underlie reliance on OCSs, including experience of distress during exacerbation, fear of future exacerbation and lack of proper knowledge about the long-term health consequences of severe asthma and OCS treatments. A multi-disciplinary approach is warranted to support the patients and to provide systematic education about the long-term health implications of severe asthma.

$@$ ERSpublications

Exacerbation widely affects the lives of people with severe asthma. Distress during exacerbation and fear of future exacerbations may underlie oral steroid reliance. Systematic patient education is warranted about the long-term health implications. https://bit.ly/3lhsBiN

Cite this article as: Song W-J, Won H-K, Lee SY, et al. Patients' experiences of asthma exacerbation and management: a qualitative study of severe asthma. ERJ Open Res 2021; 7: 00528-2020 [https:// doi.org/10.1183/23120541.00528-2020]. 


\section{Introduction}

Severe asthma is a major health concern [1]. It is estimated to affect $\sim 5-10 \%$ of the asthmatic population, and $>50 \%$ of asthma-related health costs are attributed to severe asthma [2-4]. Patients with severe asthma are prone to repeated exacerbation and progressive deterioration in lung function and may also experience side-effects from medications, such as oral corticosteroids (OCSs) [5, 6]. These acute and long-term health outcomes are considered to be the major burdens [7]. This is reflected in the European Respiratory Society (ERS)/American Thoracic Society (ATS) guidelines [5], which define severe asthma not only as asthma that remains uncontrolled despite aggressive drug therapy, but also as asthma requiring aggressive therapy to prevent it from becoming uncontrolled.

Exacerbation is a defining feature of severe asthma [5]. Exacerbation is associated with the future risk of exacerbation, leading to progressive decline in lung function and health status of patients with severe asthma [7]. In the literature, several qualitative studies have described the experience of patients with severe asthma [8-15]; however, very few studies, and none in the Asian population, have focussed on patients' experience of asthma exacerbation and the experiences that may lead to reliance on OCSs. Therefore, this qualitative study was conducted to examine the experience of Korean patients with severe asthma, with a focus on asthma exacerbation and OCS treatment.

\section{Materials and methods}

\section{Study design}

This was an inductive qualitative research, aiming to understand the experience of patients with severe asthma, with a focus on asthma exacerbation and OCS treatment. Participants were recruited between September 2018 and June 2019 from specialist clinics at three tertiary hospitals in South Korea. The patients were invited to participate if they met the following inclusion criteria: 1 ) adults ( $\geqslant 18$ years old); 2) currently having severe asthma defined according to the international ERS/ATS consensus guidelines [5]; and 3) willing to share his/her own experience. The exclusion criterion was presence of other serious psychiatric or physical illnesses, which may affect the quality of life considerably, such as malignancy, heart failure, stroke or other severe respiratory diseases. The study protocol was approved by the Institutional Review Board of each participating institution. The participants' rights were explained during the recruitment process, which is about anonymity, privacy, confidentiality, self-determination regarding their free will to participate in the study, ability to withdraw from the study and recording of the interview. All participants provided informed consent prior to the qualitative interview.

\section{Data collection}

In-depth focussed interviews were conducted by an experienced principal investigator (W.J. Joung) using open-ended questions regarding the experience of asthma exacerbation and management of patients with severe asthma. The main probing questions are summarised in table 1 . The interviews were conducted in person or via the telephone. Each interview lasted for $\sim 60-90 \mathrm{~min}$ on average. All interviews were tape-recorded and transcribed verbatim as soon as possible. Additional telephone interviews were conducted if any clarifications were required. All interviews were originally conducted and transcribed in Korean, and the findings were translated to English by the primary investigators, and later reviewed and edited by a native English speaker.

\section{Data analysis}

The method of phenomenological approach and data analysis by ColAizzi [16] was followed to gain a deeper understanding of the phenomena and to describe the phenomena as they present themselves. The main author read all the participants' descriptions several times to gain a general impression of their experiences and decipher them. Secondly, significant phrases and sentences pertaining to the experience of severe asthma were extracted, repetitions were eliminated and participants' statements were transformed

\section{TABLE 1 Main probing questions for study participants}

What is your experience of asthma worsening (or exacerbation)?

Which particular aspects of asthma exacerbation trouble you?

How do you recognise that asthma is worsening (or an asthma attack is coming on)?

How do you feel when your asthma symptoms worsen?

How do you manage your asthma when your asthma worsens (drug/non-drug)?

What are the main issues/troubles in managing asthma exacerbation?

What is your experience of drug treatments?

What problems do you experience with oral corticosteroid treatment? 
into general formulations. In the third step, the author attempted to identify the hidden meaning in each significant statement and formulated the meaning of these statements. In the fourth step, the formulated meaning was sorted into theme clusters. In the fifth step, the author integrated all of the results into a narrative description. The fundamental structure of the experience was then identified. Finally, the author performed the validation process by showing the study results to some participants to verify whether the findings accurately reflected the essence of their experience. The final number of patients was determined by content saturation.

Four criteria, i.e., credibility, fittingness, auditability and confirmability, were applied to enhance the rigour of the findings [17]. The steps of purposeful sampling, in-depth individual interviews with open-ended questions and validation of these results were taken to ensure the credibility of the results. In addition, the primary investigators regularly discussed the emerging themes in the iterative processes of data collection and analyses. To achieve fittingness, the author attempted to identify cases rich in information to the point of saturation and to describe each participant's experience in detail. Auditability was achieved by closely following the analysis steps of Colaizzi [16] and carefully documenting the research procedures. Finally, confirmability was achieved by minimising personal prejudices or biases. In addition, participants' comments were cited to help the readers confirm that the findings were derived from the patients' data.

\section{Results}

21 patients meeting the selection criteria were considered as potential candidates, and 14 patients were finally recruited. The recruitment stopped when content saturation was reached. Eight (57.1\%) patients were women, and the median (range) age was 54 years (36-70 years). Asthma duration was 17 years (5-35 years). All participants had active disease, with a median of three exacerbations and two emergency room (ER) visits/hospitalisations for asthma in the last year. All had a documented variability in airflow obstruction, and eight had fixed airflow obstruction. Chronic rhinosinusitis was comorbid in eight patients. Although we did not intend to select patients with type 2 inflammation, all had at least one sign of type 2 inflammation and were taking OCSs (nine out of 14 were taking OCS as a maintenance therapy). Total OCS dose in the last 12 months was a median (range) of $900 \mathrm{mg}$ (420-2775 mg, prednisolone equivalent). None of them was being treated with biologics at enrolment in this study. Their clinical characteristics are summarised in table 2. Four theme clusters emerged from the analyses using the method by Colaizzi [16] (figure 1).

\section{TABLE 2 Characteristics of study participants}

\begin{tabular}{|c|c|}
\hline Subjects & 14 \\
\hline Age years & $54(36-70)$ \\
\hline Female/male & $8 / 6$ \\
\hline Smoking status (never/past/current) & $10 / 4 / 0$ \\
\hline Body mass index $\mathrm{kg} \cdot \mathrm{m}^{-2}$ & $24.2(22.1-31.0)$ \\
\hline Asthma-onset age years & $37(10-55)$ \\
\hline Asthma duration years & $17(5-35)$ \\
\hline Type 2 inflammation (no/yes) ${ }^{\#}$ & $0 / 14$ \\
\hline Asthma Control Test score" & $18(12-22)$ \\
\hline $\mathrm{FEV}_{1} \%$ pred" & $68(51-89)$ \\
\hline Fixed airflow obstruction ${ }^{+}$ & 8 \\
\hline Chronic rhinosinusitis" & 8 \\
\hline Frequency of exacerbations in the last 12 months ${ }^{\pi}$ & $3(2-8)$ \\
\hline $\begin{array}{l}\text { Frequency of emergency room visits or hospitalisations for asthma exacerbation in } \\
\text { the last } 12 \text { months }{ }^{\pi}\end{array}$ & $2(0-6)$ \\
\hline \multicolumn{2}{|l|}{ Asthma treatment step" } \\
\hline GINA step 4 & 4 \\
\hline GINA step 5 & 10 \\
\hline Number of patients taking $0 \mathrm{CS}^{\pi}$ & 14 \\
\hline Taking OCS as needed & 5 \\
\hline Taking OCS maintenance & 9 \\
\hline Total OCS dose in the last 12 months $\mathbf{m g}^{\S}$ & $900(420-2775)$ \\
\hline \multicolumn{2}{|c|}{ 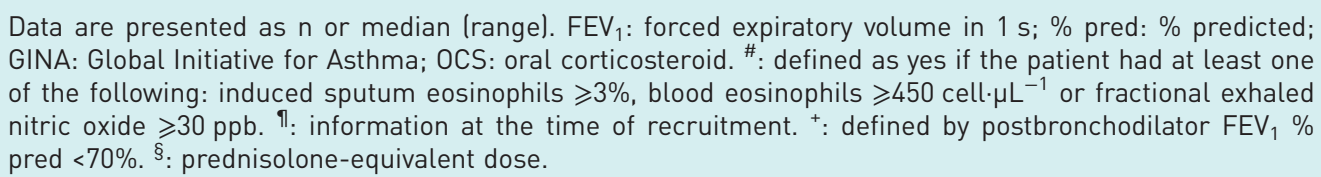 } \\
\hline
\end{tabular}




\section{Experience of exacerbations}

Distress, panic and fear of death

Feeling helpless with failed efforts

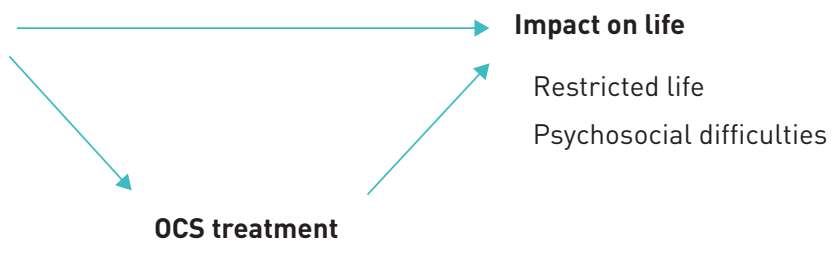

OCS reliance with mixed feelings

OCS side-effects without prior knowledge

Poor perception of severe asthma

FIGURE 1 Conceptual diagram of the overarching theme clusters regarding asthma exacerbation and experience in patients with severe asthma. OCS: oral corticosteroid.

Theme cluster 1: experience of asthma exacerbations

This theme cluster is about the experiences and feelings of patients at the moment of asthma exacerbation.

\section{Theme 1.1: distress, panic and fear of death during acute exacerbation}

The participants described severe distress during exacerbations. They commonly referred to two symptoms, breathlessness and cough, as major factors causing distress during asthma exacerbation. Some patients described "fear of death" from sudden onset of shortness of breath (table 3). Acute exacerbation was expressed as a "very lonely moment in life" that the patients should cope with by themselves, as they could not even call for help.

Cough was another major symptom during asthma worsening, which causes severe distress. Coughing also led to physical complications, including chest pain, rib fracture and voice change. Some patients reported experiencing serious anxiety and a sense of crisis with coughing attacks that were severe enough to "drain out energy" or "explode their body" (table 3 ).

Poorly controlled symptoms got worse and harder to endure at night and took away patients' night-time peace and resting. They frequently encountered difficulty in falling asleep and experienced intermittent wakening; they were unable to sleep all night because of persistent cough or breathlessness.

Theme 1.2: feeling helpless with failed efforts to prevent exacerbation

The participants commonly expressed that they "felt helpless", as they recurrently failed to prevent exacerbation, despite substantial efforts, including pharmacotherapy and avoidance of triggers (table 3). All described the "common cold" as the most dreaded event because it suddenly disrupted daily life and eventually led to asthma attacks, making their efforts in vain. Some patients experienced asthma attacks that were easily triggered by fatigue, lack of sleep, emotional stress or changes in seasons or climate. They were living in tension to avoid these triggers, but could not fully avoid them, as exposure to such triggers is inevitable.

\section{TABLE 3 Theme cluster 1: experience of asthma exacerbations}

\section{Theme 1.1: distress, panic and fear of death during acute exacerbation}

"Just felt like dying... I broke out in a cold sweat, feeling like my airways blocked by cement brick, which was hard and tight... I disliked the feeling very much and really fear that it will come again" (Patient 8)

“Coughing may tear up my chest. It may tear off my blood vessels... Fortunately it didn't yet, but I feel panic from it" (Patient 5)

"Cough drains me out. I feel depleted by coughing, sometimes for up to 10 days. When it starts, the cough lasts for at least 3 or 4 h" (Patient 9)

Theme 1.2: feeling helpless with failed efforts to prevent exacerbation

"All my efforts go for nothing when I get cold... It (common cold) is the most dreadful thing to me, as I get sick and hospitalised again" (Patient 10)

"I cannot but try very hard to maintain my condition... however, it is too bothersome that my asthma easily gets worsened... which is beyond my control" (Patient 12) 
Theme cluster 2: impact of severe asthma on life

This theme cluster concerns the impact of severe asthma on daily life and psychological well-being.

\section{Theme 2.1: living a restricted life for fear of asthma re-worsening}

Patients were living a restricted life with the fear of their asthma re-worsening. They put limits on their activities or jobs and gave up what they used to do. Some young patients found themselves being unable to "make a sprint" in life and felt they were lagging behind others. Some participants (six out of 14) quitted, changed or were considering changing their jobs due to asthma (table 4). They frequently experienced difficulties even in using public transportation, as they feared going to a crowded place and becoming infected with viruses from others. They also experienced anxiety with respect to their asthma worsening owing to the possibility of exposure to dust, smoke or chemicals in public places.

\section{Theme 2.2: psychosocial difficulties}

Most felt discouraged, frustrated and depressed that their asthma had not improved but had repeatedly worsened despite their great efforts. Some described their efforts like "turning the wheel of a vicious cycle". Some experienced severe depression from physical distress and fear of death during asthma exacerbation, fear of worsening, and restrictions in daily life and activities.

Psychosocial aspects of life were also influenced. They felt embarrassed or ashamed when symptoms got out of uncontrol, such as severe cough, in front of others. They felt their relationships with others were adversely affected, fearing that their uncontrollable coughing might bother people, or that some might want to avoid them or be reluctant to come near them (table 4).

Compromise to relationships also occurred within their families. Most had mixed feelings about their family members but could not share emotional distress with them. On the one hand, they felt sorry that they could not take their full responsibility as a spouse, parent or family member, and blamed themselves. On the other hand, they felt sad that their family members did not fully understand the difficulties and distress that they were experiencing (table 4).

\section{Theme cluster 3: OCS treatment}

This theme cluster is about the patient experience with OCS treatment. The first theme is about the reliance on OCSs, and the other is about experience of the adverse effects. There was no clear difference between patients receiving OCS maintenance treatments and those taking intermittent courses.

\section{Theme 3.1: reliance on OCSs with mixed feelings}

All had been taking OCSs for years, either regularly or intermittently. They knew that it was just a "quick-fix" or "stop-gap", but not a cure. They harboured vaguely negative views on OCSs, especially the

\section{TABLE 4 Theme cluster 2: impact of severe asthma on life}

\section{Theme 2.1: living a restricted life for the fear of asthma re-worsening}

"I always get conscious of my breathing, with suspicion that it is not normal. I feel that I lag behind others. For example, my starting point may be 70 , while others are 100. I always should put an upper limit to my activities, with the fear of asthma attacks" (Patient 7)

"I feel limited with my job. In short, I cannot make a living due to asthma. I used to work as a personal trainer at a fitness center, but my health condition didn't allow me to keep it. Then I started working at my father's chicken farm, but then my symptoms got worse... as I could not tolerate dust exposure in the place, and I don't go near there any longer" (Patient 12)

Theme 2.2: psychosocial difficulties

"I got serious mood swings. I couldn't accept these changes in my health condition. I attempted suicide several times for this. I just couldn't tolerate it, as I was previously healthy and active, really" (Patient 12)

"I feel ashamed of myself when my coughing doesn't stop in front of others... Once my asthma and coughing attack started in the subway, and I saw a woman telling her children that they should stay far away from me as I may have pulmonary tuberculosis which is contagious" (Patient 9)

"(Since my asthma got worse) I began to sleep in a different room away from my husband, as my cough disturbed his sleep. Sometimes, he tried to console me, but other times he got annoyed. I also would have been annoyed if I were him..." (Patient 9)

"I even thought of getting divorced several times due to my severe asthma condition which forced me to quit my job and caused financial problems. I have not shared the room with my wife since the worsening of asthma, especially due to coughing, and our marital intimacy has weakened. Our sexual relationship has become restricted due to my breathlessness" (Patient 12) 
TABLE 5 Theme cluster 3: oral corticosteroid (OCS) treatment

Theme 3.1: OCS reliance with mixed feelings

"I don't have faith in it (OCS), as it failed to prevent the gradual worsening of my asthma... I am taking these drugs like biting the bullet" (Patient 2)

"I became more reliant on the drug (OCS) as time went by, but without any real improvement. Symptoms rather worsened. I got more obese and less healthy" (Patient 7)

"I know that (oral) steroids are not a cure for asthma but just a quick-fix... but it is the only option for now to avoid going to the emergency room..." (Patient 8)

Theme 3.2: OCS side-effects

"I became obese but cannot do any exercise because my bone weakened. I first got Cushing's syndrome, diabetes mellitus, and then osteoporosis... As I gained much weight, I lost my pride as a woman, and feel sorry for my husband" (Patient 14)

fear of adverse reactions. Some patients stated that taking OCSs is like "biting the bullet". They had no other choice but to take OCSs because they believed there was no other alternative for controlling the acute worsening and preventing ER visits. The participants wanted to stop taking OCSs, but they did not hesitate to take them or increase the dosage of OCSs when they felt that the symptoms were worsening; their fear and anxiety regarding the worsening of their asthma and subsequent risk of hospitalisation were so great that there was no hesitation in their decision to take OCSs (table 5).

\section{Theme 3.2: OCS side-effects}

All participants experienced at least one adverse effect from OCS therapy, such as weight gain, mood swings, morphological changes, osteoporosis, diabetes mellitus, hypertension or Cushing's syndrome. Some who developed progressive weight gain or morphological changes had subsequent depression and felt that their personal relationships were compromised (table 5). However, surprisingly, most were poorly informed about the long-term side-effects of OCSs. The information about side-effects was given only after they developed specific adverse symptoms or signs.

\section{Theme cluster 4: perception of severe asthma}

This theme cluster addresses gaps in the perception of severe asthma among different patients, and between patients and physicians.

\section{Theme 4.1: gaps in disease perception among patients}

All participants met the diagnostic criteria of severe asthma, but few of them clearly recognised that they had severe asthma. Some of the patients had no insight about asthma, but a vague idea of severe or uncontrolled cold or bronchitis. They were not recognisant of the acute or long-term health impact of severe asthma. Those who lacked disease insight tended to perceive the disease mostly in terms of the symptoms. On the contrary, asthma was perceived as a fatal or seriously disabling condition by the patients whose asthma was not controlled despite treatment efforts, including OCSs (table 6).

\section{Theme 4.2: gaps between patients and health professionals}

Most participants were unfamiliar with the term "severe asthma" or the concept of asthma severity (table 6). While healthcare professionals clearly recognised the participants as having severe asthma, most patients did not have a clear knowledge or understanding about their diagnosis or the long-term health consequences. Indeed, they stated that they had no or little opportunity to get information about severe asthma. Many vaguely thought that they had poor respiratory functions or were experiencing a severe cold or bronchitis frequently.

\section{TABLE 6 Theme cluster 4: perception of severe asthma}

Theme 4.1: gaps in disease perception among patients

"I suffered from severe cold last month" (Patient 6)

"There is nothing more important than this (asthma). But I may look fine to them. Then, who will accept it as a disability?" (Patient 11)

Theme 4.2: gaps between patients and health professionals

"I have not heard about severe asthma... I guess that my doctor made the diagnosis (of severe asthma) for my severe bronchitis" (Patient 5) 


\section{Discussion}

In this study, we aimed to understand the patients' experience of asthma exacerbation and its management in a group of patients with severe disease. Four theme clusters were identified: the experience of exacerbations, impact on life, OCS treatment and disease perception. The patients experienced severe physical and emotional distress from asthma exacerbation, felt helpless because of their failed efforts to prevent exacerbation and were living a restricted life due to the fear of re-worsening of asthma and hospitalisation, which altogether appear to underlie their reliance on OCSs. Most participants wanted to avoid OCSs but relied on them, as they found no other alternatives to effectively control their symptoms and prevent ER visits. Surprisingly, most had poor knowledge and understanding of severe asthma or the concept of asthma severity. They had very little awareness of the potential long-term health consequences of severe asthma, including OCS side-effects, suggesting the urgent need for systematic patient education. Collectively, this study provides an insight into which patient experience influences OCS reliance, and which patient and disease components should be targeted to improve long-term health outcomes in patients with severe asthma.

Use of OCSs is one of the most challenging issues in the management of severe asthma. Long-term OCS use is common among severely asthmatic patients across all countries $(20 \%-70 \%)$ but is relatively more frequent among Asian patients [18-20]. OCSs are one of the few available options among conventional medicines to reduce asthma exacerbation and hospitalisations, apart from recently introduced biologics [1]. However, they are also associated with systemic side-effects or even with the risk of mortality, particularly when used repeatedly or over the long term $[6,7,21]$, as illustrated by some of our participants referring to their use as "biting the bullet" or "taking poison". In the literature, several qualitative studies addressed the experiences and concerns regarding the use of OCSs among severely asthmatic patients in Western countries $[8,10-12]$, and two studies $[8,10]$ particularly focussed on the experience of OCS burden, describing "oral steroid phobia", major side-effects and cost-benefit considerations when taking OCSs. Our findings are generally in line with those of previous reports $[8,10-12]$, in terms of the experience of side-effects and also the patients' bivalent perception of the necessity of OCSs. However, our study is a meaningful addition to the literature and also extension to Korean patients, in that it reveals several factors underlying the patient's reliance on OCSs, such as agonising experience of exacerbations, fear of future re-worsening and also importantly the lack of proper prior knowledge about the side-effects of OCSs. Another issue is that OCSs may mask asthma activity and blunt the patient's perception of disease activity, strengthening the patient's decision to rely on OCSs, potentially leading to poorer long-term health outcomes [7]. Biologics targeting type 2 pathways in severe asthma may be effective therapeutic options while reducing the OCS burden [1], but they are not widely used due to the high cost. Thus, it is critical to develop and provide detailed educational programmes regarding potential harms of OCSs and self-management strategies.

All participants were living a restricted life due to the fear of asthma re-worsening. They also suffered from emotional problems, including frustration, depression and anxiety. Six participants had to quit or change or considered changing their jobs due to asthma. These findings are in line with previous observations that severe asthma is not just "bad" asthma but a serious threat to a whole life encompassing physical, emotional and personal relationships $[11,13,22]$. The career or job issue was similarly pointed out with the theme of "the body as a hindrance" in a study of Australian patients by Foster et al. [11]. Thus, multidisciplinary approaches are warranted to help these patients, including emotional, psychological and social support. There is a general consensus among specialist physicians that multidisciplinary care should be provided in clinics for severe asthma [3, 23-26], but it is still unclear how this goal is being achieved, particularly how to provide a programme for physical and psychosocial support to the patients. Patient groups for severe asthma, where patients could meet and share experiences, may be helpful in supporting their psychosocial well-being.

Notably, our study participants had poor perception of and understanding about severe asthma. For most participants, the term or concept of "severe asthma" was not familiar. Rather, they tended to perceive their disease severity mostly by means of their symptoms, and not by long-term health effects. This finding may be partly due to the fact that some patients may be in denial of having their diagnosis of "severe" disease. However, a major reason is supposed to be the lack of proper education and information about the health implications of severe asthma, particularly the long-term health effects of the disease and OCSs.

Cough was a major symptom of acute exacerbation that greatly distressed the participants with severe asthma, physically and psychologically. It was a major factor related to psychosocial distress in daily life, as it made the patients feel embarrassed or caused others to distance themselves from the patients out of the fear of infectious disease. The findings suggest that cough is a frequently overlapping clinical trait in severe asthma but is not properly controlled by conventional asthma treatments. Indeed, the impact of cough is not well addressed in conventional assessment tools, such as the Asthma Control Test (ACT) or Asthma 
Control Questionnaire (ACQ) $[27,28]$. The ACT only refers to cough within a question asking about a group of "asthma symptoms" (wheezing, coughing, shortness of breath, chest tightness or pain) [28]. In the ACQ, cough was the 6th ranked item but was excluded due to the need to reduce the number of items [27]. The Asthma Quality of Life Questionnaire includes one item for cough [29], but it is unknown if it properly addresses cough-related morbidity. Thus, tools should be refined to capture the impact of cough in severe asthma. In therapeutics, biologics targeting type 2 inflammation may help to reduce cough in patients with severe eosinophilic asthma [30]. It is unknown whether novel treatment modalities targeting cough reflex pathways might be effective in severely asthmatic patients with cough who remain uncontrolled despite optimal anti-inflammatory therapy.

This study has several limitations. First, the results may not be generalised beyond the study population, although it is not the intention of a qualitative approach. Our findings primarily add to understanding of Korean patients' perspectives on severe asthma and frequent exacerbations, and raise awareness in healthcare professionals about the patient's point of view. However, our findings are also applicable to patients with severe asthma worldwide, as most of the themes identified, such as experience of exacerbation, impact on life or OCS issues, are commonly observed in previous studies from other countries $[8,10,11,15]$. Meanwhile, the degree of a patient's understanding of severe asthma and its future risks (theme 4) may differ by population or healthcare system, but to our knowledge, such regional difference has not been well addressed in the literature. As proper understanding of the severity concept will help to reduce OCS overuse and associated complications, further studies are warranted to identify determinants for the level of understanding and effective education strategies to improve the levels. Second, our study participants were frequent exacerbators with high exposure to OCSs, which is likely a more severe subgroup even within severely asthmatic patients. However, it is due to our purposive sampling of patients who would provide unique and rich information on the experience of exacerbations as per our main questions (table 1). Third, the sample size may appear small but is consistent with qualitative studies where the data is collected until content saturation is achieved. Finally, as the interviews were conducted in Korean, translation process might have influenced the findings, particularly colloquial expressions or proverbs. However, we believe the main themes and conclusions are unaffected by the translation process.

Using the qualitative methodology, this study described the experience of asthma exacerbation and management in patients with severe asthma in their own words and provided an insight into how the disease affects their life. Several major components were suggested to underlie reliance on OCSs, including severe distress during acute exacerbation, fear of future worsening, and lack of knowledge about long-term health consequences of severe asthma and OCS treatment. These findings indicate several components that should be targeted to improve long-term health outcomes in patients with severe asthma. Further, a systematic education programme is needed to provide information about severe asthma and its long-term clinical implications for patients.

Author contributions: W-J. Song and W.J. Joung designed the study, collected and interpreted the data, wrote the first draft of the manuscript, and critically reviewed the manuscript. W.J. Joung performed the analysis, and H-K. Won, S.Y. Lee, H-K. Park and Y.S. Cho collected and interpreted the data. K.F. Chung and L.G. Heaney interpreted the data and critically reviewed the manuscript. All authors approved the final version of the manuscript.

Conflict of interest: W-J. Song reports grants from AstraZeneca during the conduct of the study. H-K. Won has nothing to disclose. S.Y. Lee has nothing to disclose. H-K. Park has nothing to disclose. Y.S. Cho has nothing to disclose. K.F. Chung has nothing to disclose. L.G. Heaney has nothing to disclose. W.J. Joung has nothing to disclose.

Support statement: This study was supported by a research grant from the Investigator-Initiated Studies Programme of AstraZeneca. The opinions expressed in this paper are those of the authors and do not necessarily represent those of AstraZeneca. Funding information for this article has been deposited with the Crossref Funder Registry.

\section{References}

1 Holguin F, Cardet JC, Chung KF, et al. Management of severe asthma: a European Respiratory Society/American Thoracic Society guideline. Eur Respir J 2020; 55: 1900588.

2 Smith DH, Malone DC, Lawson KA, et al. A national estimate of the economic costs of asthma. Am J Respir Crit Care Med 1997; 156: 787-793.

3 Kim B-K, Park S-Y, Ban G-Y, et al. Evaluation and management of difficult-to-treat and severe asthma: an expert opinion from the Korean Academy of Asthma, Allergy and Clinical Immunology, the Working Group on Severe Asthma. Allergy Asthma Immunol Res 2019; 12: 910-933.

4 Lee E, Kim A, Ye YM, et al. Increasing prevalence and mortality of asthma with age in Korea, 2002-2015 a nationwide, population-based study. Allergy Asthma Immunol Res 2020; 12: 467-484.

5 Chung KF, Wenzel SE, Brozek JL, et al. International ERS/ATS guidelines on definition, evaluation and treatment of severe asthma. Eur Respir J 2014; 43: 343-373.

6 Sweeney J, Patterson CC, Menzies-Gow A, et al. Comorbidity in severe asthma requiring systemic corticosteroid therapy: cross-sectional data from the Optimum Patient Care Research Database and the British Thoracic Difficult Asthma Registry. Thorax 2016; 71: 339-346. 
7 Song WJ, Lee JH, Kang Y, et al. Future risks in patients with severe asthma. Allergy Asthma Immunol Res 2019; 11: 763-778.

8 Gamble J, Fitzsimons D, Lynes D, et al. Difficult asthma: people's perspectives on taking corticosteroid therapy. J Clin Nurs 2007; 16: 59-67.

9 Ross CJM, Williams BA, Low G, et al. Perceptions about self-management among people with severe asthma. J Asthma 2010; 47: 330-336.

10 Hyland ME, Whalley B, Jones RC, et al. A qualitative study of the impact of severe asthma and its treatment showing that treatment burden is neglected in existing asthma assessment scales. Qual Life Res 2015; 24: 631-639.

11 Foster JM, McDonald VM, Guo M, et al. "I have lost in every facet of my life": the hidden burden of severe asthma. Eur Respir J 2017; 50: 1700765.

12 Apps LD, Chantrell S, Majd S, et al. Patient perceptions of living with severe asthma: challenges to effective management. J Allergy Clin Immunol Pract 2019; 7: 2613-2621.

13 Holmes LJ, Yorke JA, Dutton C, et al. Sex and intimacy in people with severe asthma: a qualitative study. BMJ Open Respir Res 2019; 6: e000382.

14 Eassey D, Reddel HK, Ryan K, et al. Barriers to belonging: the need for relatedness amongst people living with severe asthma. J Asthma 2021; 58: 1-9.

15 Eassey D, Reddel HK, Ryan K, et al. The impact of severe asthma on patients' autonomy: a qualitative study. Health Expect 2019; 22: 528-536.

16 Colaizzi PF. Psychological research as the phenomenologist views it. Existential Phenomenological Alternatives for Psychology. Oxford, Oxford University Press, 1978; pp. 48-71.

17 Guba EG, Lincoln YS. Effective Evaluation: Improving the Usefulness of Evaluation Results through Responsive and Naturalistic Approaches. San Francisco, Jossey-Bass, 1981

18 van Bragt J, Adcock IM, Bel EHD, et al. Characteristics and treatment regimens across ERS SHARP severe asthma registries. Eur Respir J 2020; 55: 1901163.

19 Kim MH, Kim SH, Park SY, et al. Characteristics of adult severe refractory asthma in Korea analyzed from the Severe Asthma Registry. Allergy Asthma Immunol Res 2019; 11: 43-54.

20 Taube C, Bramlage P, Hofer A, et al. Prevalence of oral corticosteroid use in the German severe asthma population. ERJ Open Res 2019; 5: 00092-2019.

21 Lee H, Ryu J, Nam E, et al. Increased mortality in patients with corticosteroid-dependent asthma: a nationwide population-based study. Eur Respir J 2019; 54: 1900804.

22 Eassey D, Reddel HK, Foster JM, et al. “... I've said I wish I was dead, you'd be better off without me”: a systematic review of people's experiences of living with severe asthma. J Asthma 2019; 56: 311-322.

23 Kim S-H, Moon JY, Lee JH, et al. Perceptions of severe asthma and asthma-COPD overlap syndrome among specialists: a questionnaire survey. Allergy Asthma Immunol Res 2018; 10: 225-235.

24 Porsbjerg C, Ulrik C, Skjold T, et al. Nordic consensus statement on the systematic assessment and management of possible severe asthma in adults. Eur Clin Respir J 2018; 5: 1440868.

25 Maltby S, McDonald VM, Upham JW, et al. Severe asthma assessment, management and the organisation of care in Australia and New Zealand: expert forum roundtable meetings. Intern Med J 2021; 51: 169-180.

26 Lin J, Yang D, Huang M, et al. Chinese expert consensus on diagnosis and management of severe asthma. J Thorac Dis 2018; 10: 7020-7044.

27 Juniper EF, O'Byrne PM, Guyatt GH, et al. Development and validation of a questionnaire to measure asthma control. Eur Respir J 1999; 14: 902-907.

28 Nathan RA, Sorkness CA, Kosinski M, et al. Development of the asthma control test: a survey for assessing asthma control. J Allergy Clin Immunol 2004; 113: 59-65.

29 Juniper EF, Guyatt G, Epstein R, et al. Evaluation of impairment of health related quality of life in asthma: development of a questionnaire for use in clinical trials. Thorax 1992; 47: 76-83.

30 Faruqi S, Sykes DL, Crooks MG, et al. Objective assessment of cough: an early marker of response to biological therapies in asthma? Lung 2020; 198: 767-770. 